\title{
Clutter Removal in Sub-Nyquist Radar
}

\author{
Yonina C. Eldar, Fellow, IEEE, Roei Levi, and Aharon Cohen
}

\begin{abstract}
Recently, a new approach to sub-Nyquist sampling and processing in pulse-Doppler radar was introduced, based on the Xampling approach to reduced-rate sampling combined with Doppler focusing performed on the low-rate samples. This method imposes no restrictions on the transmitter, reduces both the sampling and processing rates and exhibits linear signal-to-noise ratio improvement with the number of pulses. Here we extend previous work on sub-Nyquist pulse-Doppler radar by incorporating a clutter removal algorithm operating on the sub-Nyquist samples, allowing to reject clutter modeled as a colored Gaussian random process. In particular, we show how to adapt standard clutter rejection techniques to work directly on the sub-Nyquist samples, allowing to preserve high detection rates even in the presence of strong clutter, while avoiding the need to first interpolate the samples to the Nyquist grid.
\end{abstract}

Index Terms-Clutter rejection, pulse-Doppler radar, sub-Nyquist sampling.

\section{INTRODUCTION}

$\mathbf{R}$ ADAR systems detect targets by processing echoes of a transmitted signal from objects in the vicinity of the radar. Typically, signals from targets of interest are accompanied by unwanted interfering echoes, referred to as clutter. Clutter originates from a variety of elements, including natural sources such as ground, sea and rain, as well as radar countermeasures such as chaff [1]. These echoes, which may be stronger than receiver noise and signals of interest, interfere with the detection of desired targets [2], [1].

A common approach to treat clutter is to model it as a random process with Doppler frequency that follows a colored Gaussian noise distribution [2], [1], [3], [4]. The amplitudes and delays are typically assumed to be independent identically distributed (iid) random variables. To discriminate target echoes from clutter a standard processing technique is to filter the received signal with weights that maximize the signal-to-interference (SIR) ratio, which accounts for both the clutter and the background noise. It can be shown that this method is equivalent to first whitening the received signal samples, and then performing matched-filtering with respect to a whitened pulse. This interpretation will be the basis for our approach to clutter removal in sub-Nyquist radar.

Recently, a new approach to sub-Nyquist pulse-Doppler radar [5], [6] was introduced, which assumes that the targets are

Manuscript received June 08, 2014; revised July 22, 2014; accepted August 20,2014. Date of publication August 26, 2014; date of current version September 09, 2014. The associate editor coordinating the review of this manuscript and approving it for publication was Prof. Simon Maskell.

The authors are with the Department of Electrical Engineering, Technion-Israel Institute of Technology, Haifa 32000, Israel (e-mail: yonina@ee.technion.ac.il; sroei@mail.technion.ac.il; saco@mail.technion.ac.il).

Color versions of one or more of the figures in this paper are available online at http://ieeexplore.ieee.org.

Digital Object Identifier 10.1109/LSP.2014.2351827 sparsely populated in the radar's unambiguous time-frequency region. This technique detects the targets from samples of the signal taken at sub-Nyquist rates, with rate proportional to the number of targets, rather than to the signal bandwidth. Subsequent processing is performed directly on the reduced-rate samples, avoiding interpolation to the high Nyquist grid. This framework uses Xampling for reduced-rate sampling [7], [8] combined with Doppler focusing of the low-rate samples in the frequency domain. Here we show how to extend the work of [5] by adding clutter to the received target model and developing suitable processing methods operating on the sub-Nyquist samples. The proposed clutter rejection algorithm assumes a colored Gaussian clutter model, and extends previous approaches used in traditional radar systems. In particular, it is based on whitening the sub-Nyquist samples in the frequency domain followed by Doppler focusing with respect to the whitened frequencies. We show that this method improves the SIR by as much as the number of pulses $P$.

The letter is organized as follows. In Section II we introduce our signal model and review sub-Nyquist Doppler focusing and standard clutter rejection methods. Sub-Nyquist clutter processing is developed in Section III. Simulations presented in Section IV show that the proposed clutter filtering can lead to high detection even in the presence of strong clutter.

\section{Pulse-Doppler RADAR}

\section{A. Nyquist-Based Processing}

A standard pulse-Doppler radar transmits a pulse train

$$
x_{T}(t)=\sum_{p=0}^{P-1} h(t-p \tau), \quad 0 \leq t \leq P \tau .
$$

The pulse-to-pulse delay $\tau$ is referred to as the pulse repetition interval (PRI) and the entire signal duration is called the coherent processing interval (CPI). The pulse $h(t)$ is a known time-limited baseband function with continuous-time Fourier transform (CTFT) $H(\omega)=\int_{-\infty}^{\infty} h(t) e^{-j \omega t} d t$. We assume that $H(\omega)$ has negligible energy at frequencies beyond $B_{h} / 2$.

The target scene consists of $L$ non-fluctuating point targets (Swerling-0 model), where $L$ may be unknown. The pulses reflect off the targets and propagate back to the transceiver. Each target $\ell$ is defined by a time delay $\tau_{\ell}$, proportional to the target's distance from the radar; a Doppler radial frequency $\nu_{\ell}$, proportional to the target-radar closing velocity; and a complex amplitude $\alpha_{\ell}$, proportional to the target's radar cross section and all other propagation factors. Assuming the targets are sufficiently slow and far, and that their velocity is constant during the CPI, we can write the received signal as [5]

$$
x(t)=\sum_{p=0}^{P-1} x_{p}(t),
$$


where

$$
x_{p}(t)=\sum_{\ell=0}^{L-1} \alpha_{\ell} h\left(t-\tau_{\ell}-p \tau\right) e^{-j \nu_{\ell} p \tau} .
$$

Classic radar samples the received signal at its Nyquist rate $B_{h}$ followed by digital matched filtering to create the sequences $y_{p}[n]=x_{p}[n] * \overline{h[-n]}, 0 \leq n \leq N-1$ where $N=\tau B_{h}$ (which we assume for simplicity is an integer). Doppler is then detected by performing a $P$-point Fourier transform along the pulse dimension: $z_{n}[k]=\sum_{p=0}^{P-1} y_{p}[n] e^{-j 2 \pi p k / P}$ for $0 \leq k<$ $P-1$. The frequency resolution in this step is $2 \pi / P \tau$. Stacking the vectors $\mathbf{z}_{n}$, and taking absolute value, we obtain a delayDoppler map $\mathbf{Z}=\operatorname{abs}\left[\mathbf{z}_{0} \ldots \mathbf{z}_{N-1}\right]$. The $L$ strongest points in $\mathbf{Z}$ are detected as the targets.

\section{B. Sub-Nyquist Processing}

In [5] the authors suggest a sub-Nyquist radar receiver which detects the delays and Dopplers from samples of the signal taken at rates much lower than the Nyquist rate. The basic idea is to operate on the Fourier coefficients of the received signal. Denote by $X_{p}[k]$ the Fourier series of the $p$ th frame $x_{p}(t+p \tau)$ (namely we align each of the frames to start at the same point). Then, it is easy to show that (see [5])

$$
X_{p}[k]=H[k] \sum_{\ell=0}^{L-1} \alpha_{\ell} e^{-j \nu_{\ell} p \tau} e^{-j 2 \pi k \tau_{\ell} / \tau},
$$

where $H[k]=(1 / \tau) H(2 \pi k / \tau)$ is the Fourier series of $h(t)$. We assume that low-rate samples of the signal are taken such that $X_{p}[k]$ is given only over a set of values $\mathcal{K}$ of size $K=|\mathcal{K}|$ where the rate depends on $K$. Sub-Nyquist sampling techniques that operate in the time domain and result in the desired Fourier coefficients as well as a hardware prototype implementing this approach are described in [6]. For simplicity, we limit the delays and Dopplers to the same grids assumed in standard (Nyquistbased) processing. Specifically, we assume that $\tau_{\ell} / \tau=s_{\ell} / N$ where $s_{\ell}$ is an integer satisfying $0 \leq s_{\ell} \leq N-1$, and that $\nu_{\ell} \tau=$ $2 \pi r_{\ell} / P$ where $r_{\ell}$ is an integer in the range $0 \leq r_{\ell} \leq P-1$.

The Fourier coefficients are processed via Doppler focusing, which uses target echoes from different pulses to create a single superimposed pulse focused at a particular Doppler frequency. This leads to improved Doppler resolution and enhanced signal-to-noise ratio (SNR) with respect to directly processing the coefficients $X_{p}[k]$. For each possible Doppler value $r \in[0, P-1]$, the focused coefficients are given by

$$
\Psi_{r}[k]=\sum_{p=0}^{P-1} X_{p}[k] e^{j 2 \pi r p / P}=P H[k] \sum_{\ell: r_{\ell}=r} \alpha_{\ell} e^{-j 2 \pi k \tau_{\ell} / \tau},
$$

where the last equality is proved in [5]. Our problem then reduces to recovery of the delays $\tau_{\ell}$ from $\Psi_{r}[k]$, which can be solved using standard compressed sensing tools [9], or methods for estimating sums of sinusoids; see [5].

\section{Clutter}

In practice, the received signal $r(t)$ is contaminated by clutter and noise. Thus,

$$
r(t)=x(t)+c(t)+n(t)
$$

where $x(t)$ is given by (2), $c(t)$ is the clutter signal and $n(t)$ is white noise with variance $\sigma_{n}^{2}$. The clutter is similar in its structure to $x(t)$ :

$$
c(t)=\sum_{p=0}^{P-1} \sum_{q=0}^{Q-1} \tilde{\alpha}_{q} h\left(t-\tilde{\tau}_{q}-p \tau\right) e^{-j \tilde{\nu}_{q} p \tau},
$$

with the difference that in the clutter signal we assume that $\tilde{\alpha}_{q}, \tilde{\tau}_{q}$ and $\tilde{\nu}_{q}$ are independent random variables. A popular model is to choose the amplitudes $\tilde{\alpha}_{q}$ as zero-mean iid with variance $E\left\{\left|\tilde{\alpha}_{q}\right|^{2}\right\}=a$, the delays $\tilde{\tau}_{q}$ as iid with a uniform distribution on $[0, \tau]$, and $\tilde{\nu}_{q}$ as iid Gaussian random variables with mean $\nu_{d}$ and variance $\sigma_{d}^{2}[1]$, [10].

A variety of processing techniques have been developed in order to aid the detection of targets in the presence of clutter [1], [3], [4], [11], [10]. A standard clutter rejection method is to process the received samples with a bank of Doppler filters, which are chosen to maximize the SIR. The resulting Doppler filter weights are given by $w=M^{-1} v$ where $M$ is the covariance matrix of the time samples (including both noise and clutter) and $v$ is a vector corresponding to the received samples for a target with a given Doppler frequency. This processing can be shown to be equivalent to first whitening the received samples and then filtering them with a whitened matched filter. In our clutter rejection algorithm developed below for sub-Nyquist radar, we follow a similar approach where the whitening is performed in the frequency domain.

\section{Pulse-Doppler Clutter Processing}

We begin with an analysis of the clutter statistics in the Fourier domain. We then propose whitening the frequency-domain samples followed by Doppler processing with respect to whitened frequency vectors. As we will see, in general whitening results in dependencies between the focused Doppler frequencies. Thus, instead of treating each frequency separately, we jointly process all frequencies using matrix sparsity ideas [12]. In the special case in which the covariance matrix of the clutter is circulant, the whitened Doppler focused samples remain separable, and processing can be performed on each frequency independently, as in (5).

\section{A. Statistics of the Received Signal}

As before, we write $r(t)=\sum_{p=0}^{P-1} r_{p}(t)$, where $r_{p}(t)$ is the signal $r(t)$ observed over $[p \tau,(p+1) \tau)$. Thus, $r_{p}(t)=x_{p}(t)+$ $c_{p}(t)+n_{p}(t)$ where $x_{p}(t)$ is defined by $(3), n_{p}(t)$ is white noise over $[p \tau,(p+1) \tau)$, and

$$
c_{p}(t)=\sum_{q=0}^{Q-1} \tilde{\alpha}_{q} h\left(t-\tilde{\tau}_{q}-p \tau\right) e^{-j \tilde{\nu}_{q} p \tau} .
$$

Similar to (4), the Fourier series representation of $c_{p}(t+p \tau)$ is given by

$$
C_{p}[k]=H[k] \sum_{q=0}^{Q-1} \tilde{\alpha}_{q} e^{-j 2 \pi k \tilde{\tau}_{q} / \tau} e^{-j \tilde{\nu}_{q} p \tau} .
$$

Define $R$ as the $P \times K$ matrix with $k$ th column given by $R_{p}[k], k \in \mathcal{K}$ where $R_{p}[k]$ is the $k$ th Fourier coefficient of $r_{p}(t+p \tau)$. We can express $R$ as

$$
R=F_{P} A F_{N}^{K} H+C+N=F_{P} A F_{N}^{K} H+B,
$$


where $B=C+N$, and $C, N$ are the matrices with $p k$ th elements $C_{p}[k], N_{p}[k]$. In addition, $H=\operatorname{diag}(H[k])$ is a $K \times K$ diagonal matrix, $F_{N}^{K}$ denotes $K$ rows from the $N \times N$ Fourier matrix with elements $e^{-j 2 \pi \ell k / N}$ for $k \in \mathcal{K}, F_{P}$ denotes the $P \times P$ Fourier matrix with elements $e^{-j 2 \pi \ell k / P}$, and $A$ is a $P \times K$ sparse matrix containing the values $\alpha_{\ell}$ at the $L$ indices $\left(r_{\ell}, s_{\ell}\right)$. Detecting the radar scene is equivalent to recovering $A$ from $R$. To this end, we first analyze the statistics of the matrix $B$ starting from that of $C_{p}[k]$.

Proposition 1: Let $C_{p}[k]$ be defined by (9) and denote by $R_{v}[k, m]=E\left\{C_{p}[k] \overline{C_{p+v}[m]}\right\}$. Then, $E\left\{C_{p}[k]\right\}=0$ and

$$
R_{v}[k, m]=\delta_{k m} Q a|H[k]|^{2} e^{j \nu_{d} v \tau-\frac{1}{2} \sigma_{d}^{2} v^{2} \tau^{2}} .
$$

Proof: For simplicity, throughout the proof, we omit the tilde from the variables $\alpha_{q}, \tau_{q}, \nu_{q}$.

Since $\alpha_{q}, \tau_{q}, \nu_{q}$ are independent and $E\left\{\alpha_{q}\right\}=0$, we have immediately that $E\left\{C_{p}[k]\right\}=0$. Next, from (9),

$$
\begin{aligned}
& R_{v}[k, m]=H[k] \overline{H[m]} \sum_{q, \ell=0}^{Q-1} E\left\{\alpha_{q} \overline{\alpha_{\ell}}\right\} \\
& \cdot E\left\{e^{-j 2 \pi\left(k \tau_{q}-m \tau_{\ell}\right) / \tau}\right\} E\left\{e^{-j \tau\left(\nu_{q} p-\nu_{\ell}(p+v)\right)}\right\}
\end{aligned}
$$

Using the fact that $E\left\{\alpha_{q} \overline{\alpha_{\ell}}\right\}=a \delta_{q \ell},(12)$ reduces to

$$
\begin{aligned}
R_{v}[k, m] & =a H[k] \overline{H[m]} \\
& \cdot \sum_{q=0}^{Q-1} E\left\{e^{-j 2 \pi(k-m) \tau_{q} / \tau}\right\} E\left\{e^{j \tau \nu_{q} v}\right\}
\end{aligned}
$$

Since $\tau_{q}$ is uniformly distributed on $[0, \tau]$, $E\left\{e^{-j 2 \pi(k-m) \tau_{q} / \tau}\right\}=\delta_{k m}$. Finally,

$$
E\left\{e^{j \tau \nu_{q} v}\right\}=\phi_{\nu_{d}, \sigma_{d}^{2}}(v \tau)=e^{j \nu_{d} v \tau-\frac{1}{2} \sigma_{d}^{2} v^{2} \tau^{2}}
$$

where $\phi_{\nu_{d}, \sigma_{d}^{2}}(t)$ is the characteristic function of a Gaussian random variable with mean $\nu_{d}$ and variance $\sigma_{d}^{2}$.

Proposition 2: [5] Let $N_{p}[k]$ be the Fourier series of $n_{p}(t)$. Then $N_{p}[k]$ are zero-mean iid variables with variance $\sigma_{n}^{2} / \tau$.

For simplicity, we assume below that $|H[k]|^{2}=s$ for all $k$, although the derivations can also be extended to the general case. Combining Propositions 1 and 2, it follows that the columns of $B$ in (10) are uncorrelated and identically distributed. Denote by $M$ the covariance matrix of the columns. Then, $M$ is a Toeplitz matrix with $m$ th diagonal value

$$
M(m)=s Q a e^{j \nu_{d} m \tau-\frac{1}{2} \sigma_{d}^{2} m^{2} \tau^{2}}+\frac{\sigma_{n}^{2}}{\tau} \delta[m] .
$$

\section{B. Clutter Processing}

To determine $A$ from $R$ we suggest whitening $R$ by multiplying on the left by $M^{-1 / 2}$ to yield the whitened measurements

$$
Y=M^{-1 / 2} F_{P} A F_{N}^{K} H+W=\tilde{Y}+W,
$$

where now $W$ is white noise. We then perform Doppler focusing on $Y$ with respect to whitened Fourier tones, namely, we form the matrix $\Psi=Y^{H} M^{-1 / 2} F_{P}$. From (16),

$$
\Psi=H\left(F_{N}^{K}\right)^{H} A^{H} F_{P}^{H} M^{-1} F_{P}+\tilde{W}=\tilde{\Psi}+\tilde{W},
$$

where $\tilde{W}=W^{H} M^{-1 / 2} F_{P}$.

The equations in (17) represent a sparse matrix recovery problem [12] in which the goal is to recover a sparse matrix $S$ from measurements $Y \approx B S C$ where $B$ and $C$ are arbitrary matrices. In our case, the sparse matrix is given by $S=A^{H}$. Methods for efficiently solving such problems are discussed in [12]. When $D=F_{P}^{H} M^{-1} F_{P}$ is a diagonal matrix, and ignoring the noise, (17) can be expressed as

$$
\Psi \approx H\left(F_{N}^{K}\right)^{H} S
$$

where now $S=A^{H} D$ is a sparse matrix whose sparsity pattern is the same as $A^{H}$. Therefore, recovering $S$ is equivalent to detecting the required delays and Dopplers. In particular, when $M$ is a circulant matrix, $D$ is diagonal. In this case, each column of $\Psi$ can be written as a standard sparse recovery problem from partial Fourier measurements, just as in (5). We can then solve (18) using the techniques suggested in [5].

\section{SIR Enhancement}

We now show that whitened Doppler focusing generally increases the SIR. Our derivations follow those of [5], adapted to colored noise. To analyze the SIR we consider a single target with amplitude $\alpha$, delay $t=s \tau / N$ and Doppler $\nu=2 \pi r /(P \tau)$. With this choice, we compare the SIR in $Y_{p k}$ to that of $\Psi_{k p}$ (note that $\Psi$ is obtained by Doppler processing on $Y^{H}$ which is why we exchanged the indices) for $p=r$.

Since $W$ contains zero-mean iid elements with unit variance, the SIR of $Y_{p k}$ is $\left|\tilde{Y}_{p k}\right|^{2}$. Denote by $v_{r}$ the $r$ th column of $F_{P}$, and by $u_{s}$ the $s$ th row of $F_{N}^{K}$. Then,

$$
\left|\tilde{Y}_{p k}\right|^{2}=\gamma\left|e_{p}^{T} M^{-1 / 2} v_{r} u_{s} e_{k}\right|^{2}=\gamma\left|e_{p}^{T} M^{-1 / 2} v_{r}\right|^{2},
$$

where $\gamma=|\alpha|^{2}|H[k]|^{2}, e_{p}$ is the $p$ th column of the identity, and we used the fact that $\left|u_{s} e_{k}\right|^{2}=1$.

To evaluate the SIR in $\Psi_{k p}$ we compute the signal power:

$$
\left|\tilde{\Psi}_{k p}\right|^{2}=\gamma\left|e_{p}^{T} F_{P}^{H} M^{-1} v_{r} u_{s} e_{k}\right|^{2}=\gamma\left|v_{p}^{H} M^{-1} v_{r}\right|^{2} .
$$

Since $\tilde{W}=W^{H} M^{-1 / 2} F_{P}$, and $W$ consists of zero-mean, unit variance, iid random variables,

$$
E\left\{\left|\tilde{W}_{k p}\right|^{2}\right\}=v_{p}^{H} M^{-1} v_{p} .
$$

Substituting $r=p$, the SIR after Doppler focusing is

$$
\Delta=\frac{\left|\tilde{\Psi}_{k p}\right|^{2}}{E\left\{\left|\tilde{W}_{k p}\right|^{2}\right\}}=\gamma v_{p}^{H} M^{-1} v_{p} .
$$

As $\left|e_{p}^{T} M^{-1 / 2} v_{p}\right|^{2} \leq v_{p}^{H} M^{-1} v_{p}$, the SIR after focusing can only increase. When $M$ a circulant matrix, and in particular for $M=c I$, the SIR increases by $v_{p}^{H} v_{p}=P$. 


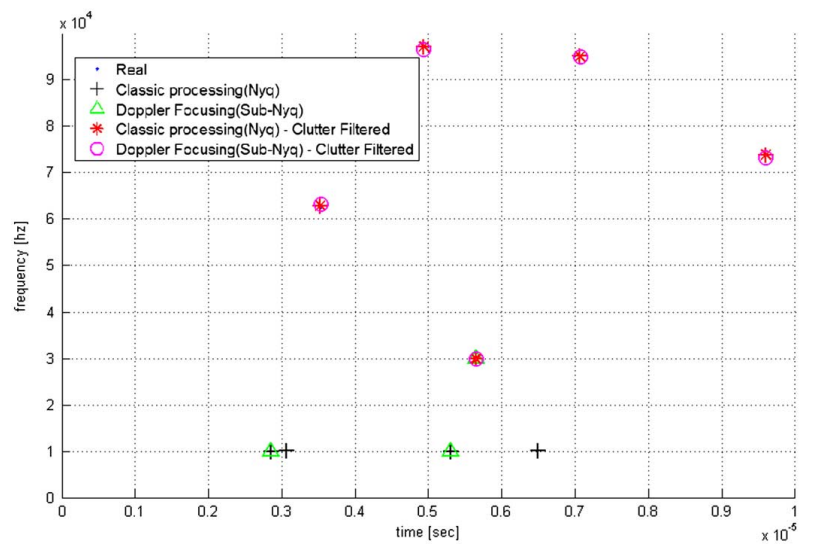

Fig. 1. Recovered target scene with 5 targets and a single clutter source, at SNR of $-25 \mathrm{~dB}$ and SCR of $-10 \mathrm{~dB}$.

\section{SIMULATIONS AND EXPERIMENT}

\section{A. Simulations}

We now present simulation results of the sub-Nyquist radar system including clutter and compare its performance to traditional Nyquist-rate radar systems. The SNR, performance metric and detection criterion used here are identical to those presented in [5]. We define the signal-to-clutter ratio (SCR) for target $\ell$ as $\left|\alpha_{\ell}\right|^{2} / \sum_{q=0}^{Q-1}\left|\tilde{\alpha}_{q}\right|^{2}$. The parameters chosen were PRI $\tau=1 \mu \mathrm{sec}$, and $B_{h}=100 \mathrm{MHz}$. Target delays and Doppler frequencies are spread uniformly at random in the appropriate unambiguous regions, and target amplitudes were chosen with constant absolute value and random phase. The unambiguous Doppler frequency interval is $[-b, b]$ where $b=\pi / \tau$.

Figure 1 illustrates target scenes and their recovery under 4 scenarios: Nyquist-rate radar processing with and without clutter rejection, sub-Nyquist processing at one tenth of the Nyquist rate with and without clutter rejection. The figure depicts the recovered target scene with 5 targets and a single clutter source with $\sigma_{d}^{2}=10^{-2} b^{2}$ and $\nu_{d}=0.1 b$. The simulation was performed under SNR of $-25 \mathrm{~dB}$, SCR of $-10 \mathrm{~dB}, P=40$ and averaging over 5 clutter targets. Without clutter filtering, both systems detect 4 false targets, due to the high clutter power. Incorporating clutter rejection, both methods recover the whole scene correctly.

In order to compare the detection rates of the two radar systems under varying conditions, we considered a target scene composed of 5 targets with $P=40$, along with one clutter source, with Doppler frequency variance $\sigma_{d}^{2}=10^{-3} b^{2}$. In Fig. 2 we plot the hit rate as a function of the SCR, for a fixed SNR of $-25 \mathrm{~dB}$. The hit rate was averaged over 20 simulation runs, such that on each run the random parameters of the targets and clutter were regenerated. This simulation shows the robustness of our clutter filtering method, achieving high detection rates at low SNR and SCR.

\section{B. Radar Experiment}

A working sub-Nyquist radar prototype employing Xampling and Doppler focusing is presented in [5], [6]. This prototype can detect targets at SNRs as low as $-25 \mathrm{~dB}$, while operating at one tenth the Nyquist rate. The same hardware was used to demonstrate clutter rejection [13], where clutter was added to the system following the model presented herein.

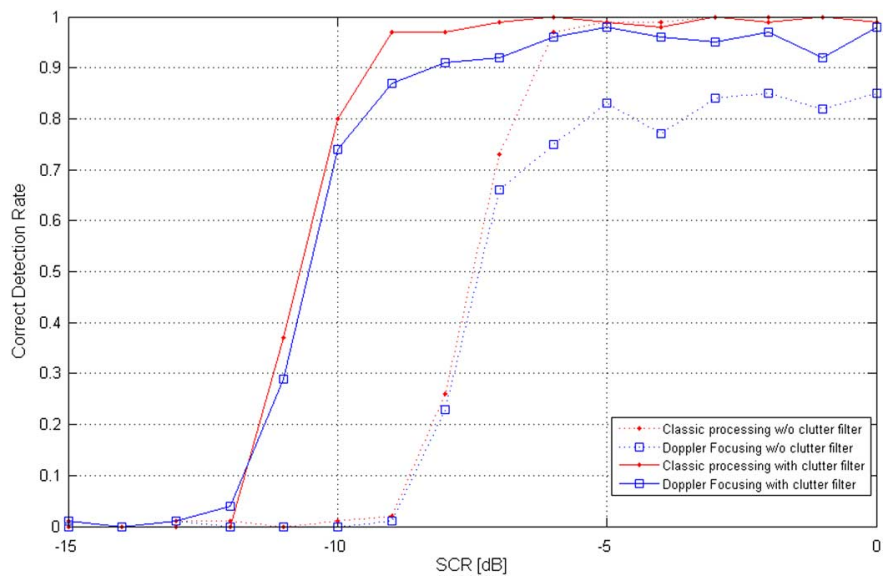

Fig. 2. Comparison of average detection rates vs. SCR at $-25 \mathrm{~dB}$ SNR, with and without clutter filtering.

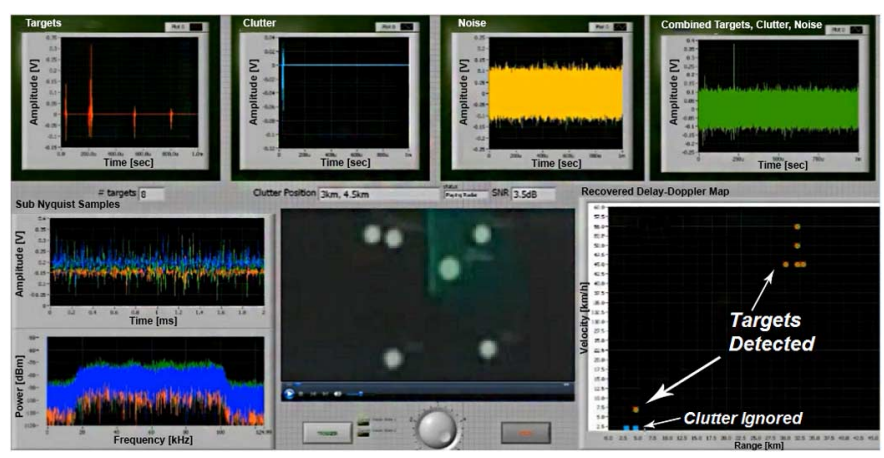

Fig. 3. The LabView-based GUI of the experimental hardware.

The GUI of the experimental hardware of [13] is shown in Fig. 3. The top views present the amplitudes in the time domain representation of the following signals (left to right): Targets, clutter, noise and all three combined. The bottom views present (left to right): Sub-Nyquist samples (time domain and frequency domain), the generated targets and the corresponding recovered delay-Doppler map. This example, which is composed of several targets at similar delays and two clutter sources, is accurately detected, while ignoring clutter. The scene consists of 6 targets; the echoes from the first 4 targets are shown in the top left window. The echoes from the clutter, shown in the top middle window, have delays very close to the first signal delay. The following two windows show that the signal and clutter are buried in the noise. The bottom left window demonstrates the effect of low-rate sampling. The low bandwidth of the sub-sampled signal is clearly evident both in time and in frequency.

\section{CONCLUSION}

We showed how to extend sub-Nyquist sampling and processing to include clutter rejection at sub-Nyquist rates by whitening and matched filtering in the frequency domain. We demonstrated clutter filtering both in simulations and on a real hardware sub-Nyquist radar prototype. We also proved that our approach increases the SIR by as much as a factor of $P$ (the number of pulses). The performance of the clutter rejection technique was compared with traditional processing methods at the Nyquist rate and was found to have comparable performance. The proposed sub-Nyquist recovery algorithm sustained SNR as low as $-30 \mathrm{~dB}$ and $\mathrm{SCR}$ as low as $-10 \mathrm{~dB}$. 


\section{REFERENCES}

[1] M. I. Skolnik, Radar Handbook. New York, NY, USA: McGraw-Hill, 2009.

[2] M. A. Richards, Fundamentals of Radar Signal Processing. New York, NY, USA: McGraw-Hill, 2005.

[3] V. Gregers Hansen, "Clutter suppression in search radars," in 1977 IEEE Conf. Decision and Control including the 16th Symp. Adaptive Processes and A Special Symposium on Fuzzy Set Theory and Applications, Dec. 1977, vol. 16, pp. 536-543.

[4] L. E. Brennan and I. S. Reed, "Theory of adaptive radar," IEEE Trans. Aerosp. Electron. Syst., vol. AES-9, no. 2, pp. 237-252, Mar. 1973.

[5] O. Bar-Ilan and Y. C. Eldar, "Sub-Nyquist radar via doppler focusing," IEEE Trans. Signal Process., vol. 62, no. 7, pp. 1796-1811, Apr. 2014.

[6] E. Baransky, G. Itzhak, I. Shmuel, N. Wagner, E. Shoshan, and Y. C. Eldar, "A sub-nyquist radar prototype: Hardware and algorithms," IEEE Trans. Aerosp. Electron. Syst., vol. 50, no. 2, pp. 809-822, Apr. 2014.

[7] M. Mishali, Y. C. Eldar, O. Dounaevsky, and E. Shoshan, "Xampling: Analog to digital at sub-Nyquist rates," IET Circuits, Devices, Syst., vol. 5, no. 1, pp. 8-20, 2011.
[8] R. Tur, K. Gedalyahu, and Y. C. Eldar, "Multichannel sampling of pulse streams at the rate of innovation," IEEE Trans. Signal Process., vol. 59, no. 4, pp. 1491-1504, Apr. 2011.

[9] Y. C. Eldar and G. Kutyniok, Compressed Sensing: Theory and Applications. Cambridge, U.K.: Cambridge Univ. Press, 2012.

[10] A. Farina and F. A. Studer, "Application of gram-schmidt algorithm to optimum radar signal processing," Proc. Inst. Elect. Eng., Commun., Radar Signal Process., vol. 131, no. 2, pp. 139-145, Apr. 1984.

[11] L. E. Brennan and I. S. Reed, "Optimum processing of unequally spaced radar pulse trains for clutter rejection," IEEE Trans. Aerosp. Electron. Syst., vol. AES-4, no. 3, pp. 474-477, May 1968.

[12] T. Wimalajeewa, Y. C. Eldar, and P. K. Varshney, "Recovery of sparse matrices via matrix sketching," arXiv preprint arXiv:1311. $2448,2013$.

[13] I. Shmuel, T. Nagar, O. Bar-Ilan, G. Ilan, A. Dikopoltsev, A. Cohen, R. Levi, E. Shoshan, and Y. C. Eldar, "A sub-Nyquist radar prototype: Hardware and software," in IEEE Int. Conf. Acoustics, Speech and Signal Processing, Florence, Italy, May 2014. 